\title{
SOFT X-RAYS FROM THE GALAXY
}

\author{
M. J. REES
}

Institute of Theoretical Astronomy, University of Cambridge, England

Below $1 \mathrm{keV}$, analyses of X-ray background data are complicated by galactic absorption effects, which cause the received intensity to vary with galactic latitude. Bowyer et al. (1968) observed that the diffuse background did not fall off as rapidly as was expected towards the galactic plane. One plausible interpretation of their data would be to suppose that a significant flux of soft X-rays emanates from the disc itself. I wish to discuss what could be inferred about the latter component from improved observations of its latitude-dependence, and by indirect methods.

Consider first an idealised model of the disc in which the absorbing interstellar matter is smooth, and the galactic X-ray emission - whatever its origin may be - is likewise smoothly distributed. The optical depth at energy $\varepsilon$ is then $\tau_{\perp}(\varepsilon) \operatorname{cosec} \theta$, where $\tau_{\perp}$ is the optical depth towards the galactic pole and $\theta$ is the galactic latitude. (At low energies, where absorption is mainly due to $\mathrm{H}$ and $\mathrm{He}, \tau_{\perp} \propto \varepsilon^{-3}$; and $\tau_{\perp} \simeq 1$ for $\varepsilon \simeq 0.3 \mathrm{keV}$.) At energies for which $\tau_{\perp}<1$, we may define an angle $\theta_{\mathrm{abs}}(\varepsilon)$ at which the optical depth would be unity. The extragalactic X-ray flux would be severely attenuated at latitudes $\theta<\theta_{\mathrm{abs}}$, and the galactic X-rays would then be relatively more important.

Absorption also affects the galactic soft X-rays, but the expected latitude-dependence of this component depends on the relative scale heights of the emission and the absorption. Suppose that the density of the interstellar gas is proportional to $f(z)$, where $z$ is the coordinate perpendicular to the plane. The precise form of $f(z)$ is uncertain, but the scale height is $\sim 100 \mathrm{pc}$. The X-ray emission per unit volume will depend differently on $z$ : let us suppose it is proportional to $f\left(k^{-1} z\right)$ (i.e. the same distribution, but a scale height $k$ times greater). At energies for which $\tau_{\perp}<1$, the intensity of this component will be roughly proportional to $\operatorname{cosec} \theta$ for $\theta>\theta_{\text {abs }}$, but for $\theta \simeq 0$ the intensity will be $k^{-1}$ times as great as for $\theta \simeq \theta_{\text {abs. }}$. Thus, if $k>1$, this component has a maximum intensity at the intermediate latitude $\theta \approx \theta_{\text {abs }}$; if $k<1$ the maximum intensity occurs in the plane. Obviously the actual emission will depend on $z$ in a manner which cannot be accurately fitted by a function $f\left(k^{-1} z\right)$. However, the important point is that, if the galactic emission is important, and has a scale height exceeding that of the gas, the diffuse $\mathrm{X}$-ray background should display a prominent maximum intensity at a galactic latitude $\theta \approx \theta_{\text {abs }}$.

The appropriate value of $k$ depends on what process is responsible for the galactic $\mathrm{X}$-rays. They may be the integrated contribution from numerous intrinsically weak sources, some of which may be observed individually by detectors with better resolution. All types of stars would have values of $k>1$, and so the maximum at $\theta \simeq \theta_{\text {abs }}$ should exist if the emission is associated with a stellar population of sources. We also expect $k>1$ if the $\mathrm{X}$-rays are due to inverse Compton scattering. One type of process 
that gives rise to emission which is more concentrated towards the plane than the absorbing material (i.e. $k<1$ ) is one which involves some interaction of nonthermal particles (whose density would presumably not increase with $z$ ) with the gas itself e.g. innerbremsstrahlung, or the line emission mechanism considered by Steigman and Silk (1969). Further investigation of the latitude-dependence may therefore indicate which type of galactic emission process is dominant. Accretion of interstellar gas by neutron stars or collapsed objects may yield a significant flux of soft X-rays, for which we would expect $k<1$.

If a substantial fraction of the diffuse X-rays in the range $0.25-0.5 \mathrm{keV}$ are of galactic origin, some limits can already be set to the emission spectrum $F(\varepsilon)$ of this component. The photons received at $\sim 0.25 \mathrm{keV}$ come effectively from distances $\$ 200$ pc, because of the absorption. If, say, $50 \%$ of the X-rays at this energy were galactic, and if this component had the same spectrum as the extragalactic background, there would be a tenfold enhancement towards the plane at energies $\gtrsim 2 \mathrm{keV}$, for which the effective pathlength may be $5-15 \mathrm{kpc}$. Since this vastly exceeds the observed galactic contribution (Cooke et al., 1969) we conclude that $F(\varepsilon)$ must fall off towards higher energies at least one power more steeply than the extragalactic spectrum. A thermal spectrum at $\sim 10^{6} \mathrm{~K}$ would be consistent with this. At even lower energies (below $0.25 \mathrm{keV}$ ) the observed radiation would be entirely galactic, and its spectrum would be $\propto \varepsilon^{3} F(\varepsilon)$, because of the more efficient absorption at lower energies. The shape of $F(\varepsilon)$ in this range is important because these very soft galactic X-rays may constitute a major heat input into the gas. Indirect evidence on the contribution of $X$-rays to the heating of interstellar matter could come from detailed studies of the ionization equilibrium. For example, because of their larger photoionization crosssections, helium and heavy elements in $\mathrm{HI}$ regions would be more highly ionized, relative to hydrogen, than would be the case if cosmic rays provided the main heat input. Such effects may be studied by means of the radio combination lines of $\mathrm{H}$ and $\mathrm{He}$, or by searching for (e.g.) NiI $\lambda 1085$ in absorption. X-rays may also modify the ionization equilibrium of interstellar $\mathrm{Ca}$ and $\mathrm{Na}$.

The cloudy and irregular nature of interstellar $\mathrm{HI}$ may prohibit any clear-cut conclusion emerging from low-resolution observations. When detailed X-ray maps are available, it will be possible to correlate them with $21-\mathrm{cm}$ data. One should then be able to decide conclusively whether the X-rays originate beyond the galactic hydrogen (in which case the X-rays would be attenuated exponentially by hydrogen clouds) or whether they originate within the disc, and to inter something about the nature and properties of discrete galactic sources.

\section{References}

Bowyer, C. S., Field, G. B., and Mack, J. E.: 1968, Nature 217, 32.

Cooke, B., Griffiths, R., and Pounds, K.: 1969, in this volume, p. 280.

Steigman, G. and Silk, J. I.: 1969, in this volume, p. 385. 